\title{
Impact-induced brittle deformation, porosity loss, and aqueous alteration in the Murchison CM chondrite
}

Romy D. Hanna ${ }^{\mathrm{a}, *}$, Richard A. Ketcham ${ }^{\mathrm{a}}$, Mike Zolensky ${ }^{\mathrm{b}}$, and Whitney Behr ${ }^{\mathrm{a}}$

a Jackson School of Geosciences, University of Texas, Austin TX 78712, USA

${ }^{\mathrm{b}}$ Astromaterials Research and Exploration Science, NASA Johnson Space Center, Houston, TX 77058, USA

*Corresponding author's e-mail: romy@jsg.utexas.edu and phone number: +1 512471 0260

Keywords: carbonaceous chondrites, chondrules, impacts, high resolution X-ray computed tomography, aqueous alteration, porosity, strain, EBSD

\begin{abstract}
X-ray computed tomographic scanning of a $44 \mathrm{~g}$ Murchison stone (USNM 5487) reveals a preferred alignment of deformed, partially altered chondrules, which define a prominent foliation and weak lineation in 3D. The presence of a lineation and evidence for a component of rotational, noncoaxial shear suggest that the deformation was caused by impact. Olivine optical extinction indicates that the sample can be classified as shock stage S1, and electron backscatter diffraction (EBSD) and electron microscopy reveal that plastic deformation within the chondrules was minimal and that brittle deformation in the form of fracturing, cataclasis, and grain boundary sliding was the dominant microstructural strain-accommodating mechanism. Textural evidence such as serpentine veins parallel to the foliation fabric and crosscutting alteration veins strongly suggest that some aqueous alteration post-dated or was contemporaneous with the deformation and that multiple episodes of fracturing and mineralization occurred. Finally, using the deformed shape of the chondrules we estimate that the strain experienced by Murchison was $17-43 \%$. This combined with the current measured porosity of Murchison suggests that the original bulk
\end{abstract}


porosity of Murchison prior to its deformation was 32.2 - 53.4\% and likely at the upper end of this range due to chondrule compressibility, providing a unique estimate of predeformation porosity for a carbonaceous chondrite. Our findings suggest that significant porosity loss, deformation, and compaction from impact can occur on chondrite parent bodies whose samples may record only a low level of shock, and that significant chondrule deformation resulting in a chondrite foliation fabric can occur primarily through brittle processes and does not require plastic deformation of grains.

\section{INTRODUCTION}

$\mathrm{CM}$ chondrites contain undifferentiated solar system material that is generally considered primitive, but detailed study has demonstrated that many chondrites have been heavily processed by deformation (impacts/burial), aqueous alteration, and brecciation (Biscoff et al., 2006; Brearley, 2006; Bunch and Chang, 1980; Stoffler et al., 1988). Unraveling these effects is important for understanding the alteration processes taking place on asteroids - for example, the cause and timing of aqueous alteration (Brearley, 2006; Krot et al., 2006) . Additionally, identifying and 'seeing through' the effects of postaccretion alteration and deformation will help better constrain models of formation of the primitive components (e.g., chondrules) as well as models of planetary accretion (e.g., location and conditions of parent body formation within the solar nebula).

Deformed chondrules and aligned, textural petrofabrics are not uncommon among chondrites (Cain et al., 1986; Dodd, 1965; Martin and Mills, 1980; Martin et al., 1975; Rubin and Swindle, 2011; Scott et al., 1992; Sneyd et al., 1988; Zolensky et al., 1997). Deformation textures have variously been attributed to burial (Cain et al., 1986; Zolensky et al., 1997) or impact (Lindgren et al., 2015; Rubin, 2012; Rubin and Swindle, 2011; Scott et al., 1992; Sneyd et al., 1988), and have been proposed to have a positive correlation with aqueous alteration among the CM chondrites (Rubin, 2012). Chondrule deformation due to impact is emerging as the favored mechanism, as several studies demonstrate a correlation between deformation and other impact-induced features such as shock level and anisotropic magnetic susceptibility (Gattacceca et al., 2005; Nakamura et al., 1992; Scott et al., 1992; Sneyd et al., 1988). In addition, experimental studies of impact compaction of chondrites show that chondrules will deform and foliate in response to impact stress 
(Nakamura et al., 1995; Nakamura et al., 2000; Tomeoka et al., 1999). It has been hypothesized that chondrule deformation during impact is due to matrix pore collapse and the chondrule 'squeezing' into the surrounding pore space (Nakamura et al., 1995; Scott et al., 1992). However, the microstructural mechanisms that allow the chondrules to change shape in such a marked degree have not been investigated in much detail. Nakamura et al. (1992) examined olivine and pyroxene in the strongly foliated Leoville CV chondrite and suggested that impact shock plastically deformed the chondrules, resulting in high dislocation densities and undulose extinction of olivine leading to its shock stage classification of S3 (Scott et al., 1992). Scott et al. (1992) found that other CVs with prominent petrofabrics also display a high shock stage $(\geq S 3)$, indicating that some plastic deformation occurred during the impact that foliated the chondrules. Based on these correlations between shock stage and petrofabric, plastic deformation of silicates within the chondrules is often assumed to be the primary strain-accommodating mechanism (Bland et al., 2014; Cain et al., 1986), but there has been no direct investigation as to whether plastic deformation is actually the mechanism by which the chondrules change shape.

Several CM chondrites also show foliated chondrules but the mechanism behind these is puzzling as CMs consistently have a low shock stage (S1-S2), suggesting that plastic deformation of the silicates within chondrules should be insignificant (Scott et al., 1992). The Murchison CM chondrite classified as S1-S2, in particular, was noted early on to have a "lineated fabric" on several stones (Fuchs et al., 1973), and Fujimura et al. (1983) found a preferred alignment of phyllosilicate basal planes (001) in their Murchison sample by using X-ray pole goniometry. More recently X-ray computed tomography (XCT) has been used to show that chondrules in Murchison are deformed and show a moderate foliation (Hanna et al., 2012; Lindgren et al., 2015). Lindgren et al. (2015) explained this apparent anomaly by proposing that Murchison, along with most other CMs, has experienced multiple mild-intensity impacts which foliated the chondrules but left the ferromagnesian silicates relatively unshocked.

Another process that has been intimately linked with impact-induced foliation fabrics in chondrites is porosity loss. An inverse correlation between porosity and impactinduced petrofabrics (anisotropy of magnetic susceptibility) has been found in ordinary chondrites (Gattacceca et al., 2005; Sugiura and Strangway, 1983) and Martin and Mills 
(1980) made the more general observation that less friable (presumably, less porous) chondrites display stronger preferred alignments of deformed chondrules. Impact experiments into the Allende $\mathrm{CV}$ chondrite verified that progressively stronger impacts increasingly reduced the porosity while increasing the $2 \mathrm{D}$ aspect ratio of deformed chondrules (Nakamura et al., 2000). All of these observations lend strong support to the idea that chondrules are deforming from impact as pore collapse in the matrix (i.e. porosity loss) is occurring (Nakamura et al., 1995; Scott et al., 1992). Therefore, quantifying the amount that chondrules have been deformed from an initial state could also provide the amount of matrix porosity loss that the chondrite has experienced.

Porosity is an important material property that greatly affects a wide range of physical processes. Porosity significantly influences cratering mechanics; not only does it contribute to the attenuation of impact shock waves but it also determines the amount and distribution of waste heat generated (Melosh, 1989; Sharp et al., 2006). Porosity affects permeability and the movement of water through an object, controlling the extent and type (e.g., open- vs. closed-system) of aqueous alteration. Porosity also influences a material's thermal conductivity and therefore its thermal inertia (Zimbelman, 1986), which has implications for the movement of heat and energy on an asteroid. Meteorites, being samples of asteroids, are expected to exhibit physical characteristics, including porosity, similar to them. However, measurement of meteorite porosity and comparison with the estimated porosity of asteroids based on remote observations has shown that asteroids are significantly more porous than their meteorite analogs. The average porosity of $\mathrm{CM}$ chondrites is $\sim 24.7 \%$ (Macke et al., 2011) whereas C-type asteroids (generally interpreted to represent $\mathrm{CM}$ chondrite material) have an average porosity of $\sim 38 \%$ with values up to $80 \%$ (Britt et al., 2002).

Typically this porosity difference is attributed to macroporosity in asteroids that is larger than the scale of our meteorite samples (Britt and Consolmagno, 2000). High macroporosities can have significant implications for the internal coherency and structure of an asteroid, leading to the interpretation of an asteroid as a 'rubble-pile' (Britt and Consolmagno, 2000). But there is also surely a sampling bias, as highly porous, friable carbonaceous chondrites are unlikely to survive ejection from the parent asteroid (Scott et al., 1992) or Earth entry and landing (Baldwin and Sheaffer, 1971; Consolmagno et al., 
2008). Indeed, carbonaceous chondrites comprise less than $\sim 2 \%$ of our meteorite collection but spectral data indicate that low-albedo carbonaceous asteroids dominate ( $>$ 75\%) the main belt asteroid population (Chapman, 1999; McSween, 1999). Therefore, our chondrite collection may not provide an accurate representation of actual asteroid microporosities.

We have two primary objectives with this work. First, we investigate the fabric of the deformed chondrules in Murchison to determine its origin and to quantify the strain the sample has undergone. We then use this information to derive an estimate of predeformation porosity. Second, we use a range of microanalytical techniques to determine the microphysical mechanisms (brittle vs. plastic) and the strain-accommodating phases that allowed the chondrules in Murchison to change shape from their initial state. This will enable us to determine the cause and conditions of deformation in the Murchison sample and explore the implications and consequences of such deformation in the larger picture of secondary processing on the CM parent body.

\section{ANALYTICAL METHODS}

\subsection{X-ray Computed Tomography}

We used X-ray computed tomography (XCT) to create digital three-dimensional (3D) datasets of Murchison sample USNM 5487 (44 g). XCT is a non-destructive imaging technique that produces a series of two-dimensional (2D) images (slices) where the gray scales in each image represent X-ray attenuation, which is to first order dependent on the density and mean atomic number $(Z)$ of the material (Ketcham and Carlson, 2001). It is completely non-destructive (no sample preparation required and the instrument does not heat or alter the sample in any way) and provides a full 3D context to textural and/or

petrographic observations. For more details on and geological applications of XCT refer to Ketcham and Carlson (2001) and Ebel and Rivers (2007).

We scanned the sample both in its entirety and within two smaller sub-volumes at higher resolution (Fig. 1). The full-volume and sub-volume \#1 scans were done on the ACTIS scanner Feinfocus subsystem (microfocal X-ray source and image intensifier detector) at the University of Texas High Resolution X-ray CT Facility (UTCT) with 
intensity control off and in high power mode. The full-volume scan settings were $120 \mathrm{kV}$, $0.25 \mathrm{~mA}, 1800$ views, 2 samples per view, inter-slice spacing $58.16 \mu \mathrm{m}$ and inter-pixel spacing $53.71 \mu \mathrm{m}$. The sub-volume scan settings were $180 \mathrm{kV}, 0.22 \mathrm{~mA}, 2400$ views, 2 samples per view, inter-slice spacing $32.05 \mu \mathrm{m}$, and inter-pixel spacing $29.30 \mu \mathrm{m}$. The specimen was mounted in a plexiglass cylinder, and the X-ray signal was calibrated by measuring the signal through the empty cylinder. Sinograms were corrected for ring artifacts, streaks, and beam hardening using in-house IDL-based software. For the fullvolume scan the beam hardening correction method described in Ketcham and Hanna (2014) was used and parameters included two-material mode, reducing image resolution by $4 \mathrm{x}$ during optimization runs, and 5 points for the linearization function. The sub-volume scan had less beam hardening, due to its simpler geometry and the higher X-ray energy used, so a standard polynomial correction was applied. Slices were reconstructed using a customized version of the standard BIR reconstruction software to produce 16bit TIFF images. A post-reconstruction rotation correction was applied to the full-volume scan to correct for stage rotation errors between slice acquisition sets. A post-reconstruction ring correction (Ketcham, 2006) was applied to the sub-volume TIFFs to eliminate remaining rings, and for both scans duplicate slices in overlapping sets were deleted.

We scanned the second sub-volume on the Xradia microXCT instrument at the UTCT facility. The sub-volume was scanned at $100 \mathrm{kV}$ and $10 \mathrm{~W}$ with a 1 second acquisition time per view (1081 views) using the LFOV objective. The detector-object distance was $43.5 \mathrm{~mm}$ and the source-object distance was $66.0 \mathrm{~mm}$. The detector pixels were binned by a factor of 2 to increase the signal-to-noise ratio and a $1 \mathrm{~mm} \mathrm{CaF}_{2}$ pre-filter used to decrease beam-hardening artifacts. Instrument stage dithering prevented ring artifacts and all other reconstruction parameters (center shift, beam-hardening correction, byte scaling and kernel smoothing) were applied in the Xradia instrument software. The final reconstructed data set has a voxel size of $40.54 \mu \mathrm{m}$.

\subsection{XCT Fabric Measurement}

XCT data from the full-volume and sub-volume \#1 scans are shown in Fig. 1 and Electronic Annex (EA) videos 1 and 2. The brightest greyscales in the images represent 
the most attenuating material (i.e. the phases with the highest density and/or mean atomic number) and the darkest greyscales the least attenuating material. We defined two components based on greyscale and morphology: a volumetrically minor bright component and a relatively more abundant dark component that is almost always manifested as an elliptical object in 2D (Fig. 1B). We segmented the bright objects (isolated from the XCT volume) using an automatic thresholding algorithm in Blob3D (Ketcham, 2005a; Ketcham, 2005b).

The dark objects required more specialized processing, as their greyscale range overlapped with that of the surrounding matrix. The only reliable means of delineating them accurately was manual segmentation in Avizo ${ }^{\mathrm{TM}}$ software, principally "brush" tools that allow one to paint the regions in the data. Hand-painting all voxels encompassing a given chondrule is very labor intensive, however, and a more efficient alternative was developed. Starting in Avizo ${ }^{\mathrm{TM}}$, one or more representative cross sections in each orthogonal plane were manually segmented for each chondrule. These data were then exported to Blob3D, and an ellipsoid was fitted to their outer margins using a specialized merit function. This partial segmentation method was tested and found to provide an accurate representation of orientation and degree of anisotropy of the object compared to the traditional full segmentation approach in Blob3D (Ketcham, 2005a). To get an accurate shape and orientation measurement, only dark objects without visible fractures were segmented for analysis.

After segmentation the size, location, and orientation information of each object was calculated using Blob3D (Ketcham, 2005a; Ketcham, 2005b). Orientation information is derived by fitting an ellipsoid to the segmented component (using either the full object or orthogonal sections) and exporting the magnitude and orientation of the three axes of the ellipsoid. These ellipsoid orientations are plotted on stereonets in lower hemisphere projection using the Stereo32 software (Roeller and Trepmann, 2010). Because the scan geometries of the two sub volumes were different from the full volume scan, the orientation measurements from the sub volumes were rotated to match the full volume scan orientation (procedure described in Appendix A).

Stereo32 is also used to test whether a set of object (ellipsoid) orientations are nonrandom and define a petrofabric using the spherical statistics parameters defined by 
Woodcock and Naylor (1983). These authors define two parameters, $K$ and $C$, which describe the shape and strength respectively of a fabric defined by a set of vector orientations (in our case, the set of ellipsoid axes). For a set of orientations, the three eigenvectors are calculated and the eigenvalues (i.e. magnitude of the eigenvectors) are defined as $S_{1}, S_{2}$, and $S_{3}$. The petrofabric shape is characterized by the shape parameter

$$
K=\ln \left(\frac{S_{1}}{S_{2}}\right) / \ln \left(\frac{S_{2}}{S_{3}}\right)
$$

which ranges from zero (girdle or 'great circle' distribution on a stereonet; $K<1$ ) to infinite (cluster distribution; $K>1$ ) (Woodcock and Naylor, 1983). The strength of the fabric is characterized by the strength parameter

$$
C=\ln \left(\frac{S_{1}}{S_{3}}\right)
$$

where a weak fabric has $C$ close to zero and a strong fabric has $C$ around 4 or above (Woodcock and Naylor, 1983).

Because the calculated eigenvectors represent the three dominant, orthogonal directions in a set of individual orientations, they also uniquely describe the fabric orientation of each scan volume. For example, the primary (longest) ellipsoid axes of uniformly flattened objects that define a foliation fabric will lie in a girdle (shape parameter $K<1$ ) when plotted on a stereonet (Turner and Weiss, 1963). The first and second calculated eigenvectors of these primary axes will lie on this great circle and the third eigenvector will be orthogonal to these, i.e. it will represent the pole to the foliation plane. For a lineation fabric, the primary ellipsoid axes will lie in a cluster (shape parameter $K>1$ ) on the stereonet and therefore the first eigenvector (strongest dominant direction) of this cluster will represent the lineation direction. Hence, in this work we define the orientation of the lineation and foliation direction in each scan volume as the orientation of the first and third eigenvectors, respectively, of the primary ellipsoid axes.

To determine the error in derived orientations of objects and fabrics using the partial orthogonal section method we tested this method on an XCT dataset of a garnetkyanite schist that had been previously measured using the full segmentation method in Blob3D (Ketcham, 2005b). We segmented the garnets using both methods (partial orthogonal sections and full segmentation) and compared the derived orientations between them. Based on our results we estimate that measurement errors in absolute axial 
orientations for individual objects using best-fit ellipsoids to orthogonal sections will be up to $9^{\circ}$ and slightly higher $\left(10-12^{\circ}\right)$ when using only 3 orthogonal planes to represent the object. However, as multiple measurements are combined to derive a fabric orientation, moderate to strong fabrics $(\mathrm{C}>2.0)$ will have only about $4^{\circ}$ of measurement error in fabric orientation (depending on the sample size and number of planes used). Weaker fabrics (C $<2.0)$ will have larger errors in fabric orientation, up to $11^{\circ}$.

\subsection{Optical and Electron Microscopy}

We used the derived foliation plane and lineation direction orientations of darktoned XCT objects in the full-volume scan to cut a thin section from the sample approximately perpendicular to the foliation and parallel to the lineation. Comparison of the actual cut thin section to the XCT data indicates that the thin section was cut approximately $101.9^{\circ}$ to the foliation plane and $7.4^{\circ}$ off of the lineation direction. The polished section was examined using an optical microscope and a JEOL JSM-64900 LV SEM in the Electron Microbeam Analysis Laboratory in the Jackson School of Geosciences at the University of Texas at Austin. The section was carbon-coated for the SEM and all images and data were collected at $15 \mathrm{kV}$ in high vacuum mode. The working distance was $10 \mathrm{~mm}$ and the spot size setting varied between 50 and 62 (unitless as it determines both the beam diameter and illumination current on this model). The smaller spot size was used for backscattered electron (BSE) imaging, as the large difference in atomic number $Z$ in the sample caused image saturation at larger spot sizes. The larger spot sizes were used for energy dispersive X-ray spectroscopy (EDS) for more efficient spectrum collection and greater accuracy in peak identification. For all EDS spectra the EDAX Genesis software was used for automatic peak identification, but all identified peaks were manually verified. EDS spectra were used for qualitative phase identification where possible and for qualitative elemental composition otherwise. EDS X-ray elemental maps were collected using the automated collection algorithm provided by the EDAX Genesis software using major element K $\alpha$ peaks.

\subsection{Electron Backscatter Diffraction}


After general petrography and phase identification was complete we removed the carbon coating and further polished the section for electron backscatter diffraction (EBSD). Carbon removal and polishing consisted of the following steps (section cleaned and rinsed between steps): 1) removal of carbon with 1 micron diamond polish, 2) polishing with colloidal silica, 3) 1 micron diamond polish, 4) 0.3 micron alumina polish, and 5) final polishing with colloidal silica. We performed EBSD on an FEI XL 30 ESEM equipped with an HKL Nordlys EBSD detector at the Electron Microbeam Analysis Laboratory in the Jackson School of Geosciences at the University of Texas at Austin. Section was mounted at a $70^{\circ}$ tilt and all patterns were acquired at 25 or $30 \mathrm{kV}$ with varying spot size 6.0-7.0 and working distance 11.0-17.9 mm. $\mathrm{H}_{2} \mathrm{O}$ gas was used to keep constant pressure between 0.1 and 0.3 Torr (varied per session). Step sizes for automated mapping varied between 0.1 and 1.2 microns per pixel. Pattern acquisition and indexing was done in Oxford AZtec 2.2 software and we used the HKL Channel 5 software suite for postprocessing and analysis.

\section{RESULTS}

\subsection{XCT Petrofabric}

Using auto-segmentation in Blob3D, we segmented light-toned objects from each of the three XCT scan data sets. To eliminate orientation biasing that can occur when an object subtends only a few voxels, any objects with a tertiary (short) ellipsoid axis less than 3 voxels in length were removed (Ketcham, 2005b). A summary of results is presented in Table 1 and the orientations of the primary and tertiary axes of the best-fit ellipsoids to the light-toned objects are presented in Fig. 2. Overall the orientations of both axes in all volumes are widely distributed, but contouring (calculated using cosine sums in the Stereo32 software) and a 99\% confidence interval for a non-random distribution suggests that a preferred orientation may be present in the full-volume and sub-volume \#1 data. However, not only are the calculated $C$ strength parameters low ( $C$ $\leq 1.1$ ), but also the shape factor $K$ is different for each dataset. The full-volume primary and tertiary axes display a weak girdle distribution ( $K=0.9$ and $K=0.8$, respectively) while 
sub-volume \#1 axes both indicate a cluster distribution $(K=2.0$ and $K=1.3)$. This fabric inconsistency between scan volumes, combined with the complete lack of fabric in the sub-volume \#2 dataset, suggests that no significant fabric is present among the light-toned inclusions and they are generally randomly orientated within the Murchison sample. We checked for evidence of a stronger fabric among subsets of inclusions in the full-volume data by limiting analysis to objects with the largest volume, highest aspect ratio (primary/tertiary axis lengths), or highest elongation (primary/secondary axis lengths), but found none.

A total of 475 dark-toned objects were segmented and measured among the three scan volumes and a summary of results is presented in Table 2. Fitted ellipsoid shapes of the dark-toned objects, plotted on a ternary diagram using the TRI-PLOT software (Graham and Midgley, 2000), show that the majority of the objects (66\% total) depart from a compact (spherical) shape and that all three scan volumes show the same spread in shape distribution with a heavier trend (39\% of all objects) toward an elongate (rod-like) shape (Fig. 3). Figure 4 plots the orientations of the ellipsoids (primary and tertiary axes) and a prominent non-random fabric is apparent, with all three scan volumes showing a preferred orientation of the object primary axes along a great circle girdle and a strong clustering of the tertiary axes, indicating that the dark-toned objects define a foliation fabric (Turner and Weiss, 1963). The $K$ shape parameter supports these interpretations, confirming a girdle distribution for the primary axes (whole $K=0.6$; sub-volume \#1 $K=0.4$; sub-volume \#2 $K=0.2$ ) and a cluster distribution for the tertiary axes (whole $K=3.4$; sub-volume \#1 $K=2.5$; sub-volume \#2 $K=5.6$ ). All six distributions are found to be non-random at the $99 \%$ confidence interval (Woodcock, 1977; Woodcock and Naylor, 1983). In addition, there is a weak clustering of the primary axes (suggested by contouring and supported by a slightly larger $K(0.6)$ for the whole volume), suggesting a subtle lineation in addition to the foliation (Turner and Weiss, 1963). The primary and secondary ellipsoid axis lengths of full-volume dark-toned objects are plotted in Fig. 5. A linear regression line through the zero intercept with a high correlation (0.91) and slope of 1.22 indicates consistent object shape elongation of about $22 \%$ in the long axis direction, further supporting the presence of a weak lineation in the Murchison sample. 
Among the three scan volumes there do appear to be differences in the derived fabric strengths and orientations. Both the full-volume and sub-volume \#1 have a moderate foliation strength with $C=2.17$ (whole) and $C=2.14$ (sub-volume \#1). Subvolume $\# 2$ has a lower $C$ value of 1.52 corresponding to a relatively weaker fabric. The differences in these fabric strengths are also readily visible in Fig. 3, with sub-volume \#2 displaying a more diffuse distribution of points around the great circle girdle. Figure 3 also suggests that the foliation orientation among the three volumes slightly differ and this is quantified in Table 3. The orientation differences between the full-volume and subvolume $\# 1$ is $11.3^{\circ}$ (foliation) and $20.2^{\circ}$ (lineation). Both volumes have moderate fabric strengths $(C>2.0)$ so the estimated measurement error is $4^{\circ}$, suggesting that a subtle orientation change may be present. Sub-volume \#2 shows a lower orientation difference from the full-volume ( $6.0^{\circ}$ for the lineation and $6.6^{\circ}$ for the foliation), and due to its weaker fabric $(C<2.0)$ this falls within the expected measurement error $\left(11^{\circ}\right)$. However, when compared directly to sub-volume \#1, sub-volume \#2 shows a much higher orientation change, suggesting again that sub-volume \#1 may have a subtle but real orientation difference compared to the rest of the sample. Finally, we checked to see if the differences in fabric strength and orientation among the data sets might result from systematic bias due to the different scanners and settings used but found no evidence of this (Appendix B).

We also plotted the size frequency distribution of the dark-toned objects segmented within each volume (Fig. 6) to investigate whether the different fabric orientations and strengths among the volumes correlated with the size of the segmented objects. Because the full-volume scan has the lowest spatial resolution, the objects segmented in this volume are biased towards larger sizes with the last bin including objects up to $10.7 \mathrm{~mm}^{3}$ in size. The full-volume size frequency distribution is still an approximate normal distribution similar to the sub-volume scans with a peak around $0.3 \mathrm{~mm}^{3}$ (equivalent spherical radius $\sim 415$ microns). More significantly however, the two sub-volume scans which showed marked departures in orientation (sub-volume \#1) and fabric strength (sub-volume \#2) from the full-volume scan have similar size frequency distributions, suggesting that these fabric differences are independent of the size distributions of the segmented objects. We do note that sub-volume $\# 2$ does include the greatest number of objects smaller than 0.1 
$\mathrm{mm}^{3}$ in size and this may contribute to a weaker measured fabric as discussed in more detail below. Finally, we found no correlation between the degree of elongation or aspect ratio and the size of the object in any of the three scan volumes.

\subsection{Identification of XCT Objects}

To identify the mineralogy and rheology of the dark- and light-toned XCT objects we examined and compared the thin section cut from the scanned sample with matching XCT data. A BSE image and EDS map of a portion of the section compared to the colocated XCT data are shown in Figure 7. X-ray attenuation is a strong positive function of the atomic number $\mathrm{Z}$ of a material (Ketcham and Carlson, 2001), and since backscattered electrons are also strongly proportional to $Z$ (Reed, 2005), is expected that the relative grayscales between the two datasets will be similar as is shown in Fig. 7. However, the significantly higher resolution of the BSE image ( $2 \mu \mathrm{m} /$ pixel) reveals finer scale compositional heterogeneity that is concealed by the lower resolution $(\sim 30 \mu \mathrm{m} / \mathrm{pixel}) \mathrm{XCT}$ data.

Seven light-toned and ten dark-toned XCT objects were examined in the section using optical petrography, BSE imaging, and EDS to determine the dominant phases composing the two populations. Six of the seven light-toned XCT objects are Fe-bearing olivine crystal fragments or porphyritic Fe-bearing olivine chondrules (Fig. 7). The other light-toned object identified is an 800-micron altered CAI containing 1-10 micron spinels rimmed with Fe-bearing serpentine. The ten dark-toned XCT objects were all Mgpyroxene and $\mathrm{Mg}$-olivine porphyritic (POP) chondrules, or fragments of POP chondrules, with minor low-Ca pyroxene and Fe-bearing phyllosilicate (Fig. 7). In several cases the Fe-bearing phyllosilicate is visible in the XCT data as bright spots within the dark-toned object interiors (Fig. 7a). The matrix, which appears brighter in XCT relative to the darktoned objects, is relatively higher in Fe due to the presence of Fe-bearing phyllosilicate and clumps of tochilinite/cronstedtite (Fig. 7c). In summary, the dominant factor separating light and dark components in the XCT data appears to be the presence of iron, with the dark-toned porphyritic chondrules being dominated by pure $\mathrm{Mg}$ silicates. 
We note that the fine-grained rims (FGRs) present around many of the chondrules in Murchison are characterized by an absence of tochilinite/cronstedtite as has been documented in other CMs (Trigo-Rodriguez et al., 2006) and therefore will appear dark in the XCT data relative to the surrounding matrix (Fig. 7c). At the scan resolution of this study (30-60 $\mu \mathrm{m}$ per voxel) the FGRs are not resolvable and therefore segmentation of the dark-toned XCT objects and subsequent size and orientation analysis includes this rim. However, Fig. 7 suggests that the shape and orientation of a chondrule may differ with and without the FGR. This 3D shape departure is currently being investigated with higher resolution XCT data as a separate study (Hanna and Ketcham, 2015).

We examined the three XCT data sets in detail for additional textural or petrographic features such as lithic clasts, veins, and fractures. We did not find any evidence of clasts or veins but fracturing is common, and in particular we found a set of 5 pervasive fracture planes running sub-parallel to the foliation plane defined by the deformed chondrules. However, some of these fracture planes emanate from desiccation cracks in the fusion crust suggesting their origin is related to heating and fragmentation during atmospheric entry. In searching for other deformation textures, we found one clear example of a dark-toned XCT object deforming around a light-toned object and confirmed that the direction of flattening suggested by the deformation is normal to the foliation plane (Fig. 8). Therefore, this texture is likely the result of the same deformation event that caused foliation of the Mg-silicate porphyritic chondrules, and also suggests the bright and dark-toned XCT objects differ not only in composition (Fe) but in rheology as well.

\subsection{Detailed Petrology and Microstructures}

We investigated the thin section optically and examined olivines, both in chondrules and in the matrix, for undulose extinction that might be indicative of shock. Out of 42 olivine grains 50 microns or larger, only six grains displayed undulose extinction and the remaining grains exhibited sharp optical extinction. Using the shock stage classification scheme of Scott et al. (1992), this classifies our Murchison sample as S1, which is consistent with other studies that have assigned Murchison as shock stage S1 to S1/S2 (Rubin, 2012; Scott et al., 1992; Tomeoka et al., 1999). Some of the chondrule 
pyroxene is clearly twinned and pyroxene also commonly poikilitically encloses smaller olivines (Fig. 9). Much of the pyroxene has a mottled appearance under crossed polars, but it is unclear at the optical scale whether this is undulose or mosaic extinction or is due to very fine twinning. Brittle crystalline fracturing is pervasive in olivine and pyroxene, both in chondrules and in the matrix (Fig. 9). In some instances these fractures appear to be filled with alteration products (serpentine) but in most cases extensively fractured olivine shows no evidence of internal alteration along fractures (Fig. 9-10).

We examined fifteen chondrules in thin section that were also measured in the XCT data to investigate mineral phases, microtextures, and alteration features, and relate them to their 3D shape and orientation derived from XCT. Using BSE images, we mapped the distribution and proportion of olivine, $\mathrm{Mg}$ pyroxene, low Ca pyroxene, serpentine (or other serpentine group phase), and other phases (sulfide and oxide). While the phase mapping and proportion estimation was necessarily done in 2D thin section, we avoided any chondrules that crossed the section on their outer edges. We tested for possible correlations between amounts of the various phases with the 3D aspect ratio (i.e. amount of flattening) or 3D elongation index (i.e. amount of lengthening) of the chondrules but found none, suggesting that the relationship between chondrule shape and phase abundance is random.

Fracturing and other brittle microtextures within and around the chondrules often show systematic relationships with the foliation plane and/or lineation direction. Fractures are commonly parallel to foliation (white arrows in Fig. 10A-C), and we found several instances where these fractures have been filled with alteration products such as $\mathrm{Mg}-\mathrm{Fe}$ serpentine (white arrows in Fig. 10C) with some veins displaying central pores indicating that the mineralization did not fill the void space completely or that the vein was still opening as secondary phases were being precipitated (black arrows in Fig. 10E). As is seen in the XCT data, textures consistent with compression perpendicular to the foliation plane are also common (Fig. 10B). Brittle fracturing of the anhydrous silicates is abundant, leading to a cataclasis-like texture in which the interior portions of the chondrules look 'crunched' (Fig. 10C-D). We did find a few cases where chondrules show evidence of less deformation and in some isolated areas preserve the texture of original mesostasis. Figure 11 displays images of a chondrule with a lower 3D aspect ratio (1.29 
compared to 1.67 for the chondrule shown in Fig. 10C-F) that shows relatively less brittle fracturing of the anhydrous silicates and preserves the original texture of mesostasis in several areas. This suggests that deformation of chondrules to form a foliation was accommodated mainly by brittle fracturing and comminution, perhaps preferentially in mesostasis where the chondrule rheology was weakest. The more compressed and fractured chondrule (Figure 10) has lost all of this original mesostasis texture. Figure 12 highlights another deformed chondrule with little evidence of original mesostasis texture that instead exhibits a complex assemblage of alteration minerals, anhydrous silicate fragments, and mineralized areas in between larger fractured olivine and pyroxene crystals.

It is also apparent from the alteration textures that some aqueous alteration postdated the deformation event and that there was more than one episode of fracturing and mineralization. The largest alteration vein in the chondrule in Fig. 10C (white arrows) is parallel to the foliation plane and lineation direction, strongly implying that it is genetically related to the fabric-causing event. The presence of interior pores in the central portions of the vein indicates (Fig. 10E) that it was opened and then filled with alteration products (in this case $\mathrm{Mg}-\mathrm{Fe}$ serpentine). Further, the vein also bisects two large olivine grains (Fig. 10 C-D), suggesting that they were energetically fractured and then filled with an alteration mineral. At higher resolution it becomes apparent that the large serpentine vein has been crosscut by another vein (white arrow in Fig. 10E), implying that the massive serpentine vein was present during the formation of the second, smaller vein. This second vein is relatively brighter in the BSE images due to a higher amount of iron (white arrow in Fig. 10F). The higher iron content, along with the presence of sulfur, indicates that the smaller vein likely contains tochilinite or perhaps very fine-grained pyrrhotite. In contrast the first, more massive vein does not contain any evidence of sulfur, suggesting that it is dominated solely by $\mathrm{Mg}$-Fe serpentine. We found several other cases of cross-cutting mineralized veins within chondrule interiors suggesting multiple episodes of fracturing and mineralization, and usually at least one of the veins is parallel to the foliation and lineation direction suggesting they are related to the deformation event which foliated and lineated the chondrules. While it is possible that reflections and refractions from a single impact event could cause crosscutting fractures, it is unclear whether the aqueous fluid 
composition could evolve during the same time frame and therefore it seems more likely that the multiple fracture sets occurred during discrete deformation events.

We also see evidence of relative movement aligned with the lineation direction, and evidence that aqueous alteration postdated the movement. Figure 13A-B shows a fragmented Fe-bearing olivine chondrule in which the movement of a major fragment is largely in the direction of the lineation. In the area between the fragments numerous needle-like fibers of serpentine have grown in all directions, including perpendicular to the compression direction indicated by the foliation plane. The absence of deformation of the serpentine needles and the fact that they have not been dehydrated suggests that Murchison has experienced little deformation or thermal metamorphism since their emplacement. Another fragmented Fe-bearing olivine chondrule is shown in Fig. 13C-F. Again, apparent movement is parallel to the lineation direction, and the movement of the fragments as well as the v-pull-apart fractures (white arrows in 13C) indicate a sinistral sense of shear (Hippertt, 1993). The v-pull-apart fracture in the smaller fragment has made pore space in the fine-grained rim for the formation of tochilinite (Fig. 13F). Additional evidence of post-deformation aqueous alteration is the deposition of tochilinite between the two chondrule fragments (Fig. 13D), although in this case the tochilinite lens is elongated parallel to the foliation and lineation direction. This could be an inherited fabric or could indicate that it was deformed during or after emplacement.

\subsection{EBSD and Plastic Deformation}

While clinopyroxene twinning can be the result of impact shock (Leroux, 2001; Rubin et al., 1997), it can also occur as a result of inversion from protoenstatite during chondrule cooling (Buseck et al., 1980). To help distinguish between the two scenarios it is necessary to determine the twinning plane and to confirm that the pyroxene is in the monoclinic form (clinoenstatite for the pure $\mathrm{Mg}$ pyroxene in our sample).

We have found using EBSD that not only is the majority of the Mg pyroxene in the form of clinoenstatite (CLEN), but that all of the 23 grains we examined are twinned on the $\{100\}$ plane, regardless of their crystallographic orientation (Fig. 14A-B). In addition, the observed twins are much larger than typical $\{100\}$ clinopyroxene shock twins $(\sim 5 \mathrm{~nm})$ 
and no $\{001\}$ shock twins were observed (Leroux, 2001). We also mapped 29 diopside grains, and while growth twins on the $\{100\}$ plane were found in several grains, no shock twins were observed. Growth twins can be distinguished from shock twins in diopside by their larger size and limitation to one or two units (Raleigh and Talbot, 1967). Element zoning patterns where visible further supported the interpretation of growth, rather than shock, twinning in diopside. For these reasons it is most likely that the pervasive CLEN twinning found in Murchison chondrules formed from inversion of protoenstatite during chondrule cooling and are not the result of shock.

However, within the twinned CLEN grains we found a weak but intriguing trend. About $20 \%$ of the twinned CLEN grains displayed prominent orthoenstatite (OEN) lamellae and these five grains, located in five different chondrules, have a preferred orientation where their $\{100\}$ crystallographic planes are approximately perpendicular to the foliation plane (i.e. direction of maximum stress) (Fig. 14C-D). While the sample size is low, stress-induced orthopyroxene lamellae have been previously reported in ureilite pigeonite (Mori and Takeda, 1988). Further, it has been well-documented that CLEN lamellae form in terrestrial orthoenstatite in the presence of shear stress (Coe and Kirby, 1975) and shock-induced CLEN lamellae have been observed in the enstatite chondrites (Rubin et al., 1997), although it is unclear if this process can occur in the reverse direction. OEN can also form from inversion of protoenstatite during cooling although it is kinetically unfavored (Buseck et al., 1980) and indeed TEM-scale intergrowths of OEN and CLEN have been observed in some enstatite chondrites (Folco and Mellini, 2000) as well as within Murchison (Martin and Lindgren, in review). However, in this case the OEN is in the form of thick $(>1 \mu \mathrm{m})$ lamellae and there appears to be a preferred orientation to grains that contain them, suggesting that their presence is related to the stress the chondrules have undergone rather than their cooling history.

We also used EBSD data to quantify the strain present in olivine and pyroxene in twelve chondrules to determine if they have been plastically deformed during the event(s) that foliated and lineated them. Plastic deformation in a crystal is manifested by an abundance of low-degree misorientations $\left(<10^{\circ}\right)$ within the crystal lattice that are indicative of dislocations resulting from stress (Brewer et al., 2009). However, very low misorientations can be at the limit of the instrument resolution, which depends on several 
factors including hardware and operating conditions (Humphreys, 2001; Prior, 1999). In interpreting correlated misorientations mapped within grains, we conservatively ignored misorientations of less than $3^{\circ}$, although including them does not change our results.

Overall we found very limited low-angle misorientations within olivine and pyroxene, but did find that pyroxene displayed a slightly higher amount of misorientation per surface area (Fig.15). This combined with the commonly sharp optical extinction of olivine and absence of shock twinning in clinopyroxene indicates that plastic deformation within chondrules was minimal and that their deformation was accommodated primarily through brittle processes.

\subsection{Porosity Loss Estimate}

Assuming that the chondrules were initially spherical and incompressible and that the strain is entirely uniaxial (coaxial, non-rotational deformation), the amount of strain or uniaxial shortening $(\varepsilon)$ can be estimated by examining the degree to which the chondrules have changed shape from a sphere to a uniformly flattened ellipsoid (Fig. 16A). After

strain $\varepsilon$ (between 0 (no shortening) and 1), the tertiary (short) axis $r_{3}$ of the deformed ellipsoid is related to the original sphere radius $R$ by

$$
r_{3}=R(1-\varepsilon)
$$

If the chondrule is incompressible then the volume remains the same requiring that

$$
R^{3}=r_{3} r_{1}^{2}
$$

where $r_{l}$ is the primary (long) axis of the deformed ellipsoid. The aspect ratio $a$ of the ellipsoid is defined by the ratio of the longest to shortest axes

$$
a=r_{1} / r_{3}
$$

Rearranging (3) and (5) to substitute for $R$ and $r_{l}$ in equation (4) and simplifying leads to

$$
\varepsilon=1-a^{-2 / 3}
$$

so the total strain $\varepsilon$ can be derived from the average aspect ratio $a$ of the deformed chondrules. Porosity $(P)$ of a material is defined as

$$
P=\left(1-\frac{V_{g}}{V_{b}}\right) \times 100 \%
$$


where $V_{g}$ is the grain volume (i.e. solid material that is not pore space) and $V_{b}$ is the bulk volume (including pore space) of the meteorite.

Because observational and experimental evidence suggests that deformation of chondrules through impact accompanies porosity loss in the surrounding matrix (Gattacceca et al., 2005; Martin and Mills, 1980; Nakamura et al., 2000; Sugiura and Strangway, 1983), we can use the strain derived using the deformed chondrules in Murchison to calculate the amount of porosity that was removed in the matrix during deformation. The form of this matrix porosity could be intergranular microporosity or fracture porosity; both would likely be removed during collapse of the matrix (pore space created from fracturing during impact is addressed in Section 4.3). If deformation of the surrounding matrix is accommodated completely by collapse of this pore space, then $V_{g}$ remains constant and after deformation the bulk volume $V_{b}$ is reduced by a factor of $(1-\varepsilon)$. Using equation (7) we can define the current (post-deformation) meteorite porosity $P_{1}$ as

$$
P_{1}=\left(1-\frac{V_{g}}{(1-\varepsilon) V_{b}}\right) \times 100 \%
$$

and the pre-compaction porosity $\left(P_{0}\right)$ can be determined by rearranging $(8)$ and substituting for $V_{g}$ into another equation (7) for $P_{0}$

$$
P_{0}=\left[1-(1-\varepsilon)\left(1-\frac{P_{1}}{100}\right)\right] \times 100 \%
$$

Therefore, the pre-deformation porosity $P_{0}$ can be determined using the total strain $\varepsilon$ and current meteorite porosity $P_{1}$.

For the full-volume XCT data set with 187 segmented chondrules, the mean aspect ratio is 1.54 with a standard deviation of 0.22 (Table 2). Using equation (6), uniaxial shortening is then 0.25 , and using the average Murchison bulk porosity of $22.1 \%$ (Macke et al., 2011) this suggests a pre-compaction porosity of $41.6 \%$ (equation (9)). Using the standard deviation of 0.22 as a proxy for the measurement and calculation error, this expands the range of uniaxial shortening to $0.17-0.31$ and the pre-compaction porosity to $35.3 \%-46.6 \%$. The highest chondrite bulk porosity measured to date is $43 \%$ for C2 Tagish Lake (Hildebrand et al., 2006), suggesting that this porosity range is reasonable for coherent chondrite rock, but is still well below the hypothesized $\sim 60-75 \%$ bulk porosity of 
original accreted material (Beitz et al., 2013; Ormel et al., 2008).

Pure uniaxial compression (non-rotational deformation) would result in a foliation fabric with uniformly flattened objects. However we do see a weak lineation in the primary axes of our chondrule best-fit ellipsoids (Fig. 4). Further, the chondrules have an elongated shape (Fig. 3 ) that is manifest as a consistent $\sim 22 \%$ elongation in the long axis compared to the intermediate axis of the chondrules (Fig. 5) and we see limited evidence for a sense of shear with some component of rotation (Fig. 13C). All of this is strong evidence for an impact, rather than burial compaction, origin for the deformation of the chondrules as the latter scenario produces pure uniaxial stress only. It is unclear how a shear component of the stress from impact would influence the porosity loss, but a conservative approach is to subtract the degree of elongation (0.22) from the calculated average aspect ratio (which assumes an isotropic shape by ignoring the secondary axis) to derive an estimate of the aspect ratio (1.32) attributed to pure uniaxial strain. This aspect ratio is equal to the lower end of our error estimate and therefore does not change the range of our porosity loss estimate. Accounting for error could lower this estimate further, but we believe this is compensated by the fact that the chondrules were at least partially compressible, as discussed below. Therefore our minimum estimate of pre-deformation porosity remains $35.3 \%$.

Another key assumption of our calculation is that the chondrules were initially spherical, which is commonly assumed in similar calculations but difficult to know for certain as these chondrules are aggregate and granular in texture (Fig. 10-11), and some chondrules are obviously fragments of originally larger chondrules (refer to Fig. 7 and 13). One test of original sphericity prior to deformation is an ellipticity plot which collates the primary and tertiary axis of each deformed object and tests for a linear fit (Ramsey and Huber, 1983). Assuming the objects were spherical and had the same rheological response to a uniaxial force, they would all plot along a line with a slope equal to their aspect ratio. Randomly orientated non-spherical shapes exposed to uniaxial compression would result in objects with a large range of aspect ratios and thus a significant amount of scatter about a linear trend. Figure 17 shows the ellipticity for 187 chondrules from the full-volume scan. Linear regression through zero intercept with a high correlation $\left(\mathrm{R}^{2}=0.86\right)$ and slope (1.52) near the mean aspect ratio (1.54) is support for nearly, but not 
absolutely, spherical chondrules prior to deformation (Ramsey and Huber, 1983). Some scatter is surely introduced by including an unknown quantity of fragmented chondrules and by the differences in rheological response among the chondrules (for example, the amount or arrangement of original mesostasis). Despite these factors the deformed chondrule shapes still show a relatively small spread in aspect ratio, suggesting that the assumption of a nearly spherical shape is reasonable.

Our calculation also assumes that the chondrules are completely incompressible and that strain occurs in 3 dimensions (contraction in $\mathrm{Z}$ and expansion in $\mathrm{X}$ and $\mathrm{Y}$ ) to produce a uniformly flattened chondrule with a constant volume. However, if the chondrules are completely compressible (i.e. contain pore space, like the matrix), strain is limited to one dimension $(Z)$, requiring a greater amount of strain in $Z$ to produce the same aspect ratio (Fig. 16B). In this case the deformed chondrule has a primary ellipsoid axis $r_{1}$ equal to the initial spherical radius $R$. Using $R$ for $\mathrm{r}_{1}$ and equation (3) for $\mathrm{r}_{3}$ in equation (5) and rearranging yields

$$
\varepsilon=1-a^{-1}
$$

Using the aspect ratio of $1.54 \pm 0.22, \varepsilon=0.24-0.43$ and results in a pre-deformation porosity of $41.0-55.7 \%$ (equation (9)).

Finally, we note that the equations describing our model of porosity collapse exclude potential complicating effects of water, notably the possible presence of ice in the pre-deformed material, and post-deformation aqueous alteration. The former concern is probably a secondary issue if we assign the ice to the pore fraction and make the simplifying assumption that it is either sparse or can be considered fully compressible due to escape during melting or vaporization. Aqueous alteration will tend to increase phase volume, and if some of this volume increase is accommodated by expansion into pore space then it may represent an additional component of porosity loss. In our model this would mean that the present-day porosity we are using is too low, and using a higher postdeformation porosity would in turn increase the inferred pre-deformation porosity.

\section{DISCUSSION}

\subsection{Brittle Deformation via Impact}


Murchison is considered a regolith breccia and therefore different regions could have experienced different deformation and alteration histories as has been seen for other CMs (Zolensky et al., 2014). We do see evidence of a subtle but real change in foliation direction and strength among the sub-volumes analyzed (Fig. 3 and Table 3 ) but the departures are not large enough to represent different clasts with markedly different histories (Friedrich et al., 2014; Zolensky et al., 2014). Overall the entire sample seems to have undergone the same deformation history resulting in a foliation signature that is relatively consistent on a cm-scale. Therefore, we interpret the foliation and lineation fabric found in our sample as resulting from in-situ deformation.

However, the subtle change in foliation and lineation orientation between subvolume \#1 and the other two scan volumes may represent a difference in in-situ finite strain within the sample. A component of rotational shear strain in Murchison is suggested by rare shear-sense indicators (Fig. 13C) and may also explain the difference in foliation and lineation orientation between the scan volumes. In the full-volume scan we found that the dark objects were consistently elongated by $22 \%$, but if we apply this same technique to sub-volume \#1 we find the objects are elongated slightly more, by $\sim 25 \%\left(\mathrm{R}^{2}=0.87\right)$. Further, subtracting this from the sub-volume \#1 average aspect ratio (Table 2) results in almost the same estimate of flattening (1.33 compared to 1.32 for the full-volume). This suggests that while the uniaxial strain between the volumes is the same, there exists a small difference in rotational strain, just as there exists a small difference in the foliation plane orientation and lineation direction between the two volumes (Table 3). This correlation between rotational strain and foliation orientation is consistent with some component of noncoaxial shear, because during noncoaxial shear, the foliation plane rotates progressively toward the shear plane (Passchier and Trouw, 2005). For coaxial, or pure shear deformation, the foliation should remain the same orientation regardless of finite strain magnitude. The cause of the difference in finite strain is difficult to determine, but is most likely the result of rheological differences on a cm-scale within the sample, related to the number or size of chondrules, proportion of matrix, or the amount of original porosity (which is likely also related to the first two properties). 
Consequently, we interpret the lineation fabric defined by the Murchison chondrules to result from a component of noncoaxial shear from impact. Lineation fabrics in chondrites have also been interpreted as an intersection fabric formed from multiple impacts (Smith et al., 2006; Sugiura and Strangway, 1983) and because we see evidence for multiple deformation events this could apply to our Murchison sample as well. However, the correlation between the foliation orientation and degree of rotational strain argues in favor of impact-induced rotational shear. Evidence for impact-induced noncoaxial shear has also been found in the Pultusk $\mathrm{H}$ chondrite in the form of shear fractures in silicates and deformed metal grains. Unlike for the Pultusk $\mathrm{H}$ chondrite however, we do not see abundant shear sense indicators nor evidence of frictional melting in localized shear zones (Krzesińska et al., 2015). Therefore, we believe that the dominant strain leading to chondrule deformation was coaxial (pure shear) compression with a minor component of noncoaxial (rotational, or simple) shear. Noncoaxial strain does occur in impact settings but the magnitude of this strain in comparison to the coaxial component (i.e. the vorticity) is unclear and is also dependent on the location within the impact structure (Melosh, 1989; Trepmann, 2008).

While the indication of noncoaxial strain is a strong argument against overburden due to burial compaction as the deformation mechanism, there is another tectonic setting other than impact that could cause simple shear. For a chondrite parent body that is partially differentiated with an overlying primitive chondritic crust (Elkins-Tanton et al., 2011; Weiss and Elkins-Tanton, 2013), it is expected that a cooling core would causing compressional thrust faulting in the overlying undifferentiated chondritic crust (ElkinsTanton, 2015) much like has been seen on Mercury (Byrne et al., 2014). While the absence of a strong impact shock signature (S1 in Murchison) could be an argument against an impact origin, there are other reasons (discussed below) why impact(s) may not impart a shock signature to chondrule silicates. The strain rate of deformation (higher for impact, lower for tectonic faulting) could be a more reliable indicator of the deformation mechanism, although this is complicated by the fact that tectonic faulting can also occur during the modification stage of sufficiently large impacts (Melosh, 1989).

In a previous study, Lindgren et al. (2015) measured a foliation fabric defined by dark-toned XCT objects (chondrules) in a smaller, separate Murchison sample using the 
method described here. The foliation fabric strength for that sample was weaker $(\mathrm{C}=1.46)$ and the average aspect ratio higher $(1.75 \pm 0.39)$ than for this sample. Unfortunately the samples are not orientated to each other so fabric orientations cannot be compared. Because we see small differences in fabric strength and average aspect ratio among sub volumes in this study that we interpret as evidence of differences of finite strain within the bulk meteorite, it is likely that the same explanation applies to the fabric difference with the other Murchison sample as well. However, because the other scan had a much higher resolution $(7.4 \mu \mathrm{m})$, dark-toned objects as small as $\sim 60 \mu \mathrm{m}$ in cross-section were included in the analysis making it more likely that some of these objects were crystal (fragments) with low X-ray attenuation (forsterite, calcite) rather than chondrules (Lindgren et al., 2015). In contrast, the smallest objects segmented for this study are $\sim 120 \mu \mathrm{m}$ in crosssection, making it less likely that this analysis includes crystal fragments. Still, sub-volume \#2 does include the greatest proportion of these smallest objects (Fig. 6) and also has a much weaker fabric than the other two scan volumes (Fig. 4) so it is possible that this population includes some large crystal fragments. Indeed, when we consider only the 77 largest objects in this scan volume, the $C$ strength of the foliation fabric increases to 1.63 (primary axes) and 2.04 (tertiary axes).

Our textural observations, most significantly a lineation and evidence for noncoaxial shear, are most consistent with impact rather than burial compaction as the deformation mechanism, in agreement with several studies that have also attributed foliation (Lindgren et al., 2015; Rubin, 2012; Scott et al., 1992; Sneyd et al., 1988) as well as lineation (Krzesińska et al., 2015) in chondrites to impact. The major outstanding question, however, is how exactly are the chondrules changing shape? Is it a plastic or brittle process? Our findings strongly suggest that plastic deformation within crystalline silicates has been minimal (Fig.15), despite the large shape change evidenced by the foliation fabric and chondrule shapes (Fig. 3-5). This is consistent with Martin and Lindgren (in review) who examined forsterite in a Murchison chondrule using TEM and found no evidence for subgrain boundaries or other defects, also indicating that plastic deformation did not occur. Despite this, we see evidence that the majority of chondrules initially had a nearly spherical shape (Fig. 17) and so significant deformation within the chondrules has taken place. Therefore, we find that the majority of this strain was 
accommodated by brittle processes, both in the form of micro-fracturing of silicates (Fig. 9-13) as well as complete fracturing of chondrules (Fig. 13). Some grain boundary sliding and structural rearrangement is certainly taking place along with fracturing, and indeed we see clear evidence of relative movement between large fragments (Fig. 13).

However, we believe the largest movement and compression is most likely taking place between the crystal silicates in the mesostasis. Chondrule mesostasis, being glass and crystallites (or aqueously altered versions of them), would be the most structurally weak material within a chondrule and would preferentially experience the most strain. We believe we see evidence of this preferential deformation of mesostasis, as preserved original mesostasis texture is rare in our Murchison sample. Most commonly between the larger olivines and pyroxene crystals we see massive, texture-less serpentine sometimes with angular fragments of pyroxene that do not have the euhedral appearance of late stage crystallization (Fig. 10-12). We interpret the massive serpentine found within and around the olivine and pyroxene as the comminuted, altered remnants of mesostasis glass. In addition, where we do see heavily fractured and fragmented silicates within or around massive serpentine, it is more commonly pyroxene than olivine (Fig. 12). This is perhaps not surprising as the pyroxene shows a degraded texture with pore space that makes it stand out relative to the olivine in BSE images despite their similar greyscale (Fig. 10-12). This degraded texture and pore space would make pyroxene weaker relative to olivine, and indeed this degraded, porous texture in pyroxene is seen in several CMs, including relatively unaltered ones, suggesting that it is present very early in CM chondrite alteration history (Hanowski and Brearley, 2001; Lindgren and Lee, 2015; Martin and Lindgren, in review). Previous workers have interpreted the preferential cracking and pore creation in CLEN to be a result of the inversion process from protoenstatite to CLEN upon chondrule cooling (Hanowski and Brearley, 2001; Yasuda et al., 1983), further suggesting that this pyroxene pore space is likely present at the time of deformation.

We therefore hypothesize that the majority of the overall shape change of the chondrule is being accommodating by fracturing, cataclasis, and perhaps collapse, firstly of the mesostasis and secondarily of pyroxene. Fracturing and cataclasis would lead to general communition of the mesostasis material and enable easier movement of it relative to the fractured, but still coherent, silicate grains. And the preferentially fragmented 
pyroxene would also contribute some movement and reorganization of the chondrule interior. We also see evidence for multiple episodes of fracturing and alteration, so the earliest alteration phases may also be preferentially accommodating some deformation (Fig. 13C-D) and allowing easier relative movement between grains (Fig. 12). Finally, some compression (volume change) may be also taking place within the chondrules if micropores are present within the mesostasis or pyroxene and being crushed out similar to void space removal in the matrix. We do see evidence of voids within altered mesostasis (Fig. 18) and pores have been found in altered mesostasis in another CM (Lindgren and Lee, 2015). We note that this implies that the chondrules are not completely incompressible as initially assumed, driving the pre-deformation porosity to higher values (section 3.5).

A natural question following this line of reasoning is why are the chondrules deforming only brittlely in Murchison, and not plastically, as impact shock is known to cause plastic deformation in silicates and this is commonly seen in other foliated chondrites (Nakamura et al., 1992; Scott et al., 1992)? It is possible that Murchison may have seen higher shock levels, but that the most highly deformed minerals have been preferentially destroyed by subsequent aqueous alteration. However, this would imply that the most deformed chondrules (i.e. with a higher aspect ratio or more elongation) would have a higher proportion of alteration products, and we do not see this correlation. Lindgren et al. (2015) addressed this paradox in Murchison by proposing that it experienced multiple, mild impacts, and our evidence of primarily brittle chondrule deformation with multiple fracturing events supports this interpretation as well. However, recent modeling of chondrule/matrix mixtures also suggests that the high initial porosity of the matrix leads to heterogeneous shock effects where the matrix experiences the highest shock pressures and temperatures while the chondrules remain relatively 'cold' (Bland et al., 2014). Therefore, the mild impact signature recorded by the Murchison chondrules could be at least partially the result of inherent material properties (i.e. porosity) and not a true reflection of the absolute impact intensity (impact velocity, pressure) experienced by the chondrite body.

Regardless, in Murchison, a relatively porous CM chondrite, impact force resulted in significant deformation of chondrules in a brittle manner and a record of high intensity 
impact leading to large amounts of plastic deformation is not found. While it is possible that annealing could have taken place after deformation and erased evidence of plastic deformation, we do not see evidence of this such as $120^{\circ}$ triple junctions between grains. In addition, the high abundance of undehydrated serpentine and tochilinite strongly suggests that Murchison was never heated to the higher temperatures required for annealing. Regardless, we do see evidence of some limited plastic deformation, mainly in clinopyroxene, which may indicate that clinopyroxene is weaker than olivine at high stresses (Raterron and Jaoul, 1991). The higher amount of plastic deformation within pyroxene could also be the result of lattice dislocations caused during the inversion from protoenstatite (Ruzicka, 1990), however this process cannot account for the higher amount of misorientations that we also see in diopside relative to olivine (Fig. 15). Regardless, the most significant portion of deformation and foliation of the chondrules is accommodated by brittle mechanisms, including some collapse of the chondrule porosity. Future models of chondrite deformation, especially when attempting to reproduce foliated or deformed chondrules, should account for this behavior instead of or in addition to plastic deformation of crystalline silicates to account for the change in shape of the chondrules (e.g. Bland et al., 2014).

Finally, we consider the implications of our observation that predominantly pyroxene and mesostasis within the Murchison chondrules are allowing the chondrules to change shape in response to the impact stress. This suggests that chondrules with a higher initial amount of these phases might allow for a larger amount of strain and shape change. As previously stated we did check for a positive correlation between amount of pyroxene or serpentine (altered mesostasis) and the shape of the chondrules and found none. However, if chondrule porosity collapses during the deformation, volume change will occur preferentially for these phases, erasing the evidence of their higher initial proportions and essentially 'normalizing out' the relative proportions of these phases among chondrules. Further, if certain chondrite classes have higher proportions of POP chondrules with abundant pyroxene and mesostasis, this might pre-disposition them to flatten and foliate in response to the presence of impact stress. In other words, the differences in petrofabric degree or strength between the different chondrite classes or 
within the classes themselves may not only reflect differences in impact intensity or conditions, but depend also on the initial rheology of the chondrules within them.

\subsection{Relative Timing of Aqueous alteration}

Our observations of microtextures involving alteration strongly suggest that some aqueous alteration post-dated or was contemporaneous with the impact events that foliated the chondrules. Several fractures parallel to the foliation fabric are filled with alteration products (Fig. 10C) with incomplete mineralization in some cases (Fig. 10E), and there is also evidence of serpentine and tochilinite precipitation after movement of chondrule fragments where the movement is also aligned with the macroscopic deformation fabric (Fig. 13). From a relative timing perspective, this seems to support the Rubin (2012) hypothesis that impacts facilitated some aqueous alteration of the CM chondrites by opening fractures within the chondrite body to allow for fluid flow. While Murchison has a relatively low degree of aqueous alteration (2.5 according to Rubin et al. (2007)) and a moderately strong foliation fabric (this work), Rubin (2012) generally found among the CMs a positive correlation between degree of aqueous alteration and petrofabric. Our Murchison sample suggests that while this direct correlation trend may not be valid for all $\mathrm{CMs}$, there is evidence of impact facilitation of aqueous alteration in even the least altered $\mathrm{CM}$ chondrites such as Murchison. Further, we find evidence that there were multiple episodes of fracturing and aqueous alteration, and that later alteration involved formation of tochilinite (or pyrrhotite) while earlier alteration did not (Fig. 10D-F).

We can also surmise that no significant deformation or heating occurred after the latest phase of aqueous alteration. First, as is common for most CMs, the presence of tochilinite and serpentine argue against significant post-formation heating as both phases dehydrate at relatively low temperature $\left(\sim 245^{\circ} \mathrm{C}\right.$ for tochilinite and $\sim 400^{\circ} \mathrm{C}$ for serpentine, depending on composition) (Fuchs et al., 1973; Tonui et al., 2014). In addition, Murchison shock experiments have shown that by $10 \mathrm{GPa}, \mathrm{Mg}$-Fe serpentine begins to decompose and tochilinite has completely decomposed (Tomioka et al., 2007). We see abundant fibrous tochilinite, and in one clear example, delicate serpentine fibers up to 40 microns long have remained undeformed and undehydrated after their emplacement 
following the major deformation event (Fig. 13). If any significant deformation or shock equivalent to what had originally ruptured the iron-bearing olivine chondrule had occurred, the serpentine fibers would have been destroyed as sheet silicates are among the first silicates to break down in the presence of shock (Horz and Quaide, 1973). This is consistent with other work using bulk $\mathrm{H}, \mathrm{C}$, and $\mathrm{N}$ abundances and isotopic compositions which also indicated that Murchison has not been heated since aqueous alteration (Alexander et al., 2013).

While some aqueous alteration certainly post-dated the impact event(s), what remains unclear is whether any aqueous alteration also pre-dated it, especially in regards to hydration of chondrule glassy mesostasis or the matrix. It is possible, although we don't see any clear evidence for it, that glassy mesostasis was hydrated prior to the impact event(s). If the chondrule mesostasis was altered prior to deformation, it would have caused some mesostasis to be microporous as the crystallites preferentially leached out (Lindgren and Lee, 2015). This would result in greater compressibility of the mesostasis and the chondrule in general, and also allow for easier grain rearrangement (grain boundary sliding) within the chondrule due to the lubricating nature of the phyllosilcates. Since hydration of glassy mesostasis represents one of the very first stages of alteration among the CMs (Rubin et al., 2007) and unaltered, glassy mesostasis is rare even in the least altered CMs (Lindgren and Lee, 2015), we favor the interpretation that mesostasis was hydrated early, if not before the first deformational event then shortly thereafter and therefore provided some degree of chondrule compressibility during later deformation event(s).

\subsection{Strain Estimate and Porosity Loss}

Using X-ray pole figure goniometry to determine the alignment of matrix serpentine basal planes, Fujimura (1983) estimated that Murchison had experienced $\sim 9 \%$ uniaxial strain. While this is slightly lower than our minimum estimate of uniaxial strain (17\%) using deformed chondrules, it is likely that their strain measurement is not directly comparable to ours. First as we have found within our single Murchison sample, different regions of Murchison may have experienced different finite amounts of strain. Second, 
other work has suggested that the strain measurement derived using phyllosilicate grain foliation may not reflect the overall strain experienced by the sample and is therefore not directly comparable to strain estimates using other sample characteristics (Etheridge and Oertel, 1979; Oertel, 1983).

As mentioned in section 3.5, a key assumption of our strain estimate is that the chondrules were initially spherical. The ellipticity analysis of the deformed chondrules (Fig. 17) suggests that the chondrule were nearly, but not perfectly, spherical. We note that with this method initial sphericity is indistinguishable from a situation in which all objects have the same non-spherical, ellipsoidal shape and have a uniform orientation. This could be the result from an earlier deformation event but it would be impossible to distinguish this from the latest deformation and therefore our strain estimate remains the same and represents the total, finite strain. The other possibility is that the chondrules were initially non-spherical and accreted with a fabric on the parent body. Perhaps a foliated texture could be produced by accretion onto the parent body but a lineated texture (Fig. 4) would be hard to explain using current models of accretion of chondrule-sized objects that predict clumping under self gravity in a weakly turbulent nebula (e.g., Cuzzi et al., 2008). Regardless, we hypothesize that although initial sphericity of chondrules is a sufficient first-order approximation for our strain calculation, the original, pre-deformation shape of POP chondrules found in Murchison was most likely not perfectly spherical and XCT study of an unbrecciated and undeformed CM (e.g., Yamato 791198 (Metzler et al., 1992)) would be required to investigate original POP chondrule shape.

We calculate that our Murchison sample had a pre-deformation bulk porosity of 35.3 to $55.7 \%$, depending on the influence of shear strain on pore compaction and the compressibility of the chondrules. The latter factor in particular is an important consideration, as we see evidence (pyroxene and mesostasis pore space) for some degree of chondrule compressibility, which means the pre-deformation porosity is likely at the higher end of this range. Regardless of these factors, however, our estimate represents a significant loss in bulk porosity on the CM parent body as a result of impact, consistent with experimental and modeling studies (Bland et al., 2014; Nakamura et al., 2000). However, impacts are also hypothesized to create porosity in the form of open fractures and granular microcracks, which might be important in facilitating aqueous alteration of 
CM chondrites (DeCarli et al., 2001; Rubin, 2012). Indeed, we see abundant evidence of microcracks and fractures within chondrules (Fig. 10-13). Therefore, obviously some porosity is being created during deformation and this is leading to a localized increase in porosity. However, an unknown portion of this porosity is later filled by aqueous alteration products (e.g., Fig. 10), complicating the estimate of porosity increase through fracturing. Therefore the remaining unfilled porosity that is created from the result of impact deformation is unknown. One study which examined the nature of porosity in ordinary chondrites using XCT found that the most compacted samples had the majority of their porosity in the form of intragranular cracks and larger fractures as a result of impact shock rather than original intergranular porosity (Friedrich and Rivers, 2013). Using the average porosity of these compacted samples $(3.9 \%)$ as an estimate of post-deformation porosity created via impact, this suggests that $18.3 \%$ of the total $22.2 \%$ current porosity of Murchison (Macke et al., 2011) is in the form of uncompacted porosity, resulting in a revised estimate of $32.2-53.4 \%$ for initial bulk porosity. Therefore, at a minimum the initial bulk porosity of Murchison was $32.2 \%$ prior to the deformation that foliated the chondrules within it, although evidence of chondrule compressibility suggests that the initial porosity was at the higher end of this range.

\section{CONCLUSIONS}

We have found that foliated and lineated chondrules within CM Murchison were deformed by impact and that primarily brittle, rather than plastic, mechanisms allowed for the deformation of the chondrules. Pyroxene and chondrule mesostasis were the primary phases accommodating the strain within chondrules, and there is evidence to suggest that the chondrules were not completely incompressible and that some pore collapse occurred within them in addition to in the surrounding microporous matrix during impact. Aqueous alteration post-dated, or was perhaps contemporaneous with the deformation, and there were multiple episodes of fracturing and mineralization. We estimate that the strain experienced by our Murchison sample was 0.17 to 0.43 and likely the upper limit of this as the chondrules were compressible along with the matrix. This composes the first estimate 
of pre-deformation bulk porosity for a chondrite, $32.2-53.4 \%$, perhaps providing a glimpse of primary, accretionary porosity for chondrite parent bodies.

\section{ACKNOWLEDGEMENTS}

We would like to express our sincere gratitude to the Smithsonian National Museum of Natural History, curator Tim McCoy, and collection manager Linda Welzenbach for the loan of Murchison USNM 5487 and for authorizing and providing new section cuts for this project. We would like to thank Associate Editor Eric Quirico, Alan Rubin, and an anonymous reviewer for thoughtful comments that greatly improved this manuscript. Thanks to James Martinez (JSC) for assistance with EBSD and section polishing, to Travis Clow and Zoe Yin (Univ. Texas at Austin) for assistance in Avizo ${ }^{\mathrm{TM}}$ segmentation and testing, and to John Hanna for help with the generation of Figure 16. Thanks to Paula Lindgren, Jon Friedrich, Martin Lee, Alex Ruzicka and Lindy ElkinsTanton for helpful discussions regarding a variety of topics related to the paper. R.D.H. is supported by the NASA Earth and Space Sciences Fellowship Program - Grant NNX13AO64H and portions of this work were funded through an Analytical Fees grant from the Jackson School of Geosciences to R.D.H. M.E.Z. was supported by a grant from the NASA Cosmochemistry Program.

\section{APPENDIX A}

Because the orientation of the sample was different for each of the three scans, it was necessary to rotate the orientation results of the two sub-volume scans to match the full-volume scan to facilitate fabric orientation comparisons. There are various ways to solve this problem but the method described here is specific to Avizo ${ }^{\mathrm{TM}} 8.0$ and the Blob3D program (Ketcham, 2005a; Ketcham, 2005b). First, relative transformations between volumes are determined in Avizo $^{\mathrm{TM}}$ using manual feature alignment. For our study we created exterior isosurfaces for the three datasets and manually translated and rotated the two sub-volumes to match the orientation of the full-volume scan using the Transform Editor. The resulting transformation vectors and angles can be accessed in Avizo $^{\mathrm{TM}}$ via the Transformation Editor Dialog (Absolute tab). Although the sub-volumes were translated to match the full-volume, only the rotation part of the transformation is 
needed to properly adjust the orientation data and therefore only the rotation vector and angle $(\theta)$ are recorded for each sub-volume. With the rotation vector normalized to a unit vector $u, v, w$ the rotation-only transformation matrix is calculated as

$$
T=\left[\begin{array}{ccc}
u^{2}+\left(v^{2}+w^{2}\right) \cos \theta & u v(1-\cos \theta)-w \sin \theta & u w(1-\cos \theta)+v \sin \theta \\
u v(1-\cos \theta)+w \sin \theta & v^{2}+\left(u^{2}+w^{2}\right) \cos \theta & v w(1-\cos \theta)-u \sin \theta \\
u w(1-\cos \theta)-v \sin \theta & u w(1-\cos \theta)+u \sin \theta & w^{2}+\left(u^{2}+v^{2}\right) \cos \theta
\end{array}\right]
$$

where $\theta$ is in radians. This matrix is then used to transform a set of orientation direction cosine vectors $\boldsymbol{a}$ to the new coordinate system $\boldsymbol{b}$ with

$$
\boldsymbol{b}=T \boldsymbol{a}
$$

However, because Blob3D and Avizo ${ }^{\mathrm{TM}}$ place the origin at different locations within a TIFF image (Avizo ${ }^{\mathrm{TM}}$ places the origin at the top left of the image and Blob3D places it at the bottom left) the y axis between their coordinate systems is flipped, and therefore the $y$ direction cosines from Blob3D are negated before and after the transform to assure that the transform is applied in the Avizo ${ }^{\mathrm{TM}}$ coordinate system.

\section{APPENDIX B}

To verify that the differences in fabric strength or orientation were not the result of systematic bias due to the different scanners and settings used, we examined five darktoned XCT objects in each sub-volume that were also segmented in the full-volume (the two sub-volumes do not overlap so it is not possible to compare them directly). We compared the shape and orientation of each of the ten objects among the three different scan volumes. Size and shape results are presented in tables B.1-B.2. For all ellipsoid axis lengths, the average measurement departures between the full-volume and sub-volume scans were much less than a single voxel size and the percent difference was at most $4.1 \%$. For the shape parameters (aspect ratio, elongation, and flatness) the percent differences were even smaller at a maximum of 3\%. Objects were consistently, slightly larger when measured in the sub-volume scans but this is unsurprising as the data resolution is higher in these scans leading to a more accurate measurement of the actual object size. For a lower resolution scan, the partial volume effect (blur) will lead to a higher-than-expected greyscale value in the outer margin voxels of a low-attenuation object as the surrounding bright matrix is incorporated in these voxels. Therefore, the outermost edge of the object will not be included in the measurement of the object and lead to small, albeit measureable, 
differences in the length scale. This propagates to the volume calculation where object volumes are on average $7-9 \%$ smaller in the lower resolution, full-volume scan. More importantly however these differences are essentially erased when calculating the shape

parameters based on relative axis lengths and therefore these match within $3 \%$ between the different datasets.

Tables B.1 and B.2 also list the angular separation between the best-fit ellipsoid orientations and these are visualized in Figure B.1. Individual axis orientation differences can be up to $26.6^{\circ}$ but the median departures between the different scans are a maximum of $14.2^{\circ}$. This is only slightly above the estimated measurement error $\left(9-12^{\circ}\right)$ for object orientation using the orthogonal plane segmentation method. More significantly, the direction of the angular departures are random in nature (Figure B1) and are therefore likely the result of the departures in general object measurement outlined above rather than different scanner geometries or settings. In summary, we do not find any systematic differences in the derived size or orientation of dark-toned XCT objects between the various scans and therefore interpret any fabric strength or orientation differences (above the measurement error) among the scan volumes to be real.

\section{REFERENCES}

Alexander C. M. O. D., Howard K. T., Bowden R. and Fogel M. L. (2013) The classification of $\mathrm{CM}$ and $\mathrm{CR}$ chondrites using bulk $\mathrm{H}, \mathrm{C}$ and $\mathrm{N}$ abundances and isotopic compositions. Geochim. Cosmoch. Acta 123, 244-260.

Baldwin B. and Sheaffer Y. (1971) Ablation and breakup of large meteoroids during atmospheric entry. J. Geophys. Res. 76, 4653-4668.

Beitz E., Blum J., Mathieu R., Pack A. and Hezel D. C. (2013) Experimental Investigation of the nebular formation of chondrule rims and the formation of chondite parent bodies. Geochim. Cosmoch. Acta 116, 41-51. 
Biscoff A., Scott E. R. D., Metzler K. and Goodrich C. A. (2006) Nature and Origins of Meteoritic Breccias. In Meteorites and the Early Solar System II (eds. D. Lauretta and H.Y.J. McSween). University of Arizona Press, Tucson. pp. 679-712.

Bland P. A., Collins G. S., Davison T. M., Abreu N. M., Ciesla F. J., Muxworthy A. R. and Moore J. (2014) Pressure-temperature evolution of primordial solar system solids during impact-induced compaction. Nature Communications 5, 5451.

Brearley A. J. (2006) The action of water. In Meteorites and the Early Solar System II (eds. D. Lauretta and H.Y.J. McSween). University of Arizona Press, Tucson. pp. 584-624.

Brewer L. N., Field D. P. and Merriman C. C. (2009) Mapping and Assessing Plastic Deformation using EBSD. In Electron Backscatter Diffraction in Materials Science (eds. A.J. Schwartz, M. Kumar, B.L. Adams and D.P. Field), 2nd ed. Springer Science+Business Media, LLC, New York. pp. 251-262.

Britt D. T. and Consolmagno G. (2000) The Porosity of Dark Meteorites and the Structure of Low-Albedo Asteroids. Icarus 146, 213-219.

Britt D. T., Yeomans D., Housen K. and Consolmagno G. (2002) Asteroid Density, Porosity, and Structure. In Asteroids III (eds. W.F.J. Bottke, A. Cellino, P. Paolicchi and R.P. Binzel). The University of Arizona Press, Tucson. p. 785.

Bunch T. E. and Chang S. (1980) Carbonaceous chondrites. II - Carbonaceous chondrite phyllosilicates and light element geochemistry as indicators of parent body processes and surface conditions. Geochim. Cosmoch. Acta 44, 1543-1577.

Buseck P. R., Nord G. L. and Veblen D. R. (1980) Subsolidus phenomena in pyroxenes. In Reviews in Mineralogy 7: Pyroxenes (ed. C.T. Prewitt). Mineralogical Society of America, Washington D.C. pp. 117-221.

Byrne P. K., Klimczak C., Celal Sengor A. M., Solomon S. C., Watters T. R. and Hauck I. I. S. A. (2014) Mercury's global contraction much greater than earlier estimates. Nature Geosci 7, 301-307. 
Cain P. M., McSween H. Y. and Woodward N. B. (1986) Structural deformation of the Leoville chondrite. Earth Planet. Sci. Lett. 77, 165-175.

Chapman C. R. (1999) Asteroids. In The New Solar System (eds. J.K. Beatty, C.C.

Peterson and A. Chaikin), 4 ed. Sky Publishing Corporation, Cambridge. pp. 337-350.

Coe R. S. and Kirby S. H. (1975) The orthoenstatite to clinoenstatite transformation by shearing and reversion by annealing: mechanism and potential applications. Contributions to Mineralogy and Petrology 52, 29-55.

Consolmagno G., Britt D. and Macke R. (2008) The significance of meteorite density and porosity. Chemie der Erde / Geochemistry 68, 1-29.

Cuzzi J. N., Hogan R. C. and Shariff K. (2008) Toward Planetesimals: Dense Chondrule Clumps in the Protoplanetary Nebula. The Astrophysical Journal 687, 1432.

DeCarli P. S., Bowden E. and Seaman L. (2001) Shock-induced compaction and porosity in meteorites. 64th Annual Meteoritical Society Meeting. Vatican City. \#5171(abstr.).

Dodd R. T., Jr. (1965) Preferred Orientation of Chondrules in Chondrites. Icarus 4, 308.

Ebel D. S. and Rivers M. L. (2007) Meteorite 3-D synchrotron microtomography: Methods and Applications. Meteoritics \& Planet. Sci. 42, 1627-1646.

Elkins-Tanton L. T. (2015). Personal Communication.

Elkins-Tanton L. T., Weiss B. P. and Zuber M. T. (2011) Chondrites as samples of differentiated planetesimals. Earth Planet. Sci. Lett. 305, 1-10.

Etheridge M. A. and Oertel G. (1979) Strain measurements from phyllosilicate preferred orientation - A precautionary note. Tectonophysics 60, 170-120.

Folco L. and Mellini M. (2000) Enstatite chemical composition and microstructures in the La Villa H4 chondrite. Meteoritics \& Planet. Sci. 35, 733-742. 
Friedrich J. M. and Rivers M. L. (2013) Three-dimensional imaging of ordinary chondrite microporosity at $2.6 \mathrm{~lm}$ resolution. Geochim. Cosmoch. Acta 116, 63-70.

Friedrich J. M., Weisberg M. K. and Rivers M. L. (2014) Multiple impact events recorded in the NWA $7298 \mathrm{H}$ chondrite breccia and the dynamical evolution of an ordinary chondrite asteroid. Earth Planet. Sci. Lett. 394, 13-19.

Fuchs L. H., Olsen E. and Jensen K. J. (1973) Mineralogy, mineral-chemistry, and composition of the Murchison (C2) meteorite. Smithsonian Contributions to Earth Sciences 10, 39.

Fujimura A., Kato M. and Kumazawa M. (1983) Preferred orientation of phyllosilicate (001) in matrix of Murchison meteorite and possible mechanisms of generating the oriented texture in chondrites. Earth Planet. Sci. Lett. 66, 25-32.

Gattacceca J., Rochette P., Denise M., Consolmagno G. and Folco L. (2005) An impact origin for the foliation of chondrites [rapid communication]. Earth Planet. Sci. Lett. 234, 351-368.

Graham D. J. and Midgley N. G. (2000) Graphical representation of particle shape using triangular diagrams: an Excel spreadsheet method. Earth Surface Processes and Landforms 25, 1473-1477.

Hanna R. D. and Ketcham R. A. (2015) 3D Measurement of fine-grained rims in CM Murchison using XCT. Met. Soc. Meeting LXXVIII. Berkeley, California. \#5350(abstr.).

Hanna R. D., Ketcham R. A. and Hamilton V. E. (2012) Inclusion Foliation in Murchison as Revealed by High Resolution X-Ray CT. Lunar Planet. Sci. XLIII. Lunar Planet. Inst., Houston. \#1242(abstr.).

Hanowski N. P. and Brearley A. J. (2001) Aqueous alteration of chondrules in the CM carbonaceous chondrite, Allan Hills 81002: implications for parent body alteration. Geochim. Cosmoch. Acta 65, 495-518. 
Hildebrand A. R., McCausland P. J. A., Brown P. G., Longstaffe F. J., Russell S. D. J., Tagliaferri E., Wacker J. F. and Mazur M. J. (2006) The fall and recovery of the Tagish Lake meteorite. Meteoritics \& Planet. Sci. 41, 407-431.

Hippertt J. F. M. (1993) 'V'-pull-apart microstructures: a new shear-sense indicator. Journal of Structural Geology 15, 1393-1403.

Horz F. and Quaide W. L. (1973) Debye-Scherrer investigations of experimentally shocked silicates. The Moon 6, 45-82.

Humphreys F. J. (2001) Grain and subgrain characterisation by electron backscatter diffraction. Journal of Materials Science 36, 3833-3854.

Ketcham R. A. (2005a) Computational methods for quantitative analysis of threedimensional features in geological specimens. Geosphere 1, 32-41.

Ketcham R. A. (2005b) Three-dimensional grain fabric measurements using highresolution X-ray computed tomography. Journal of Structural Geology 27, 1217-1228.

Ketcham R. A. (2006) New algorithms for ring artifact removal. Developments in X-Ray Tomography $V$. San Diego, CA.

Ketcham R. A. and Carlson W. D. (2001) Acquisition, optimization and interpretation of $\mathrm{X}$-ray computed tomographic imagery; applications to the geosciences. Comp. \& Geosci. 27, 381-400.

Ketcham R. A. and Hanna R. D. (2014) Beam hardening correction for X-ray computed tomography of heterogeneous natural materials. Computers \& Geosciences 67, 49-61.

Krot A. N., Hutcheon I. D., Brearley A. J., Pravdivtseva O. V., Petaev M. and Hohenberg C. M. (2006) Timescales and Settings for Alteration of Chondritic Meteorites. In Meteorites and the Early Solar System II (eds. D. Lauretta and H.Y.J. McSween). The Univeristy of Arizona Press, Tucson. pp. 525-553. 
Krzesińska A., Gattacceca J., Friedrich J. M. and Rochette P. (2015) Impact-related noncoaxial deformation in the Pułtusk H chondrite inferred from petrofabric analysis. Meteoritics \& Planetary Science 50, 401-417.

Leroux H. (2001) Microstructural shock signatures of major minerals in meteorites. European Journal of Mineralogy 13, 253-272.

Lindgren P., Hanna R. D., Dobson K. J., Tomkinson T. and Lee M. R. (2015) The paradox between low shock-stage and evidence for compaction in CM carbonaceous chondrites explained by multiple low-intensity impacts. Geochim. Cosmoch. Acta 148, 159-178.

Lindgren P. and Lee M. R. (2015) Tracking the earliest stages of aqueous alteration in the mildly altered CM chondrite EET 96029. Lunar Planet. Sci. XLVI. \#1760(abstr.).

Macke R. J., Consolmagno G. J. and Britt D. T. (2011) Density, porosity, and magnetic susceptibility of carbonaceous chondrites. Meteoritics \& Planet. Sci. 46, 1842-1862.

Martin L. and Lindgren P. (in review) Serpentinization of chondrules from the Murchison CM2 carbonaceous chondrite: replacement, cementation, and the genesis of polyhedral phyllosilicate crystals. Meteoritics \& Planet. Sci. Ms. ID MAPS-2374.

Martin P. M. and Mills A. A. (1980) Preferred chondrule orientations in meteorites. Earth Planet. Sci. Lett. 51, 18-25.

Martin P. M., Mills A. A. and Walker E. (1975) Preferential orientation in four C3 chondritic meteorites. Nature 257, 37.

McSween H. Y. (1999) Meteorites. In The New Solar System (eds. J.K. Beatty, C.C. Peterson and A. Chaikin), 4th ed. Sky Publishing Corporation, Cambridge. pp. 351-363.

Melosh H. J. (1989) Impact Cratering: A geologic process. Oxford University Press, New York.

Metzler K., Bischoff A. and Stoeffler D. (1992) Accretionary dust mantles in CM chondrites - Evidence for solar nebula processes. Geochim. Cosmoch. Acta 56, 2873-2897. 
Mori H. and Takeda H. (1988) Stress induced transformation of pigeonites from achondritic meteorites. Physics and Chemistry of Minerals 15, 252-259.

Nakamura T., Tomeoka K., Sekine T. and Takeda H. (1995) Impact-induced chondrule flattening in the Allende CV3 carbonaceous chondrite: Shock experiments. Meteoritics 30, 344.

Nakamura T., Tomeoka K., Takaoka N., Sekine T. and Takeda H. (2000) Impact-Induced Textural Changes of CV Carbonaceous Chondrites: Experimental Reproduction. Icarus 146, 289-300.

Nakamura T., Tomeoka K. and Takeda H. (1992) Shock effects of the Leoville CV carbonaceous chondrite - A transmission electron microscope study. Earth Planet. Sci. Lett. 114, 159-170.

Oertel G. (1983) The relationship of strain and preferred orientation of phyllosilicate grains in rocks - A review. Tectonophysics 100, 413-447.

Ormel C. W., Cuzzi J. N. and Tielens A. G. G. M. (2008) Co-accretion of chondrules and dust in the solar nebula. The Astrophysical Journal 679, 1588-1610.

Passchier C. W. and Trouw R. A. J. (2005) Microtectonics, 2nd ed. Springer, Berlin.

Prior D. J. (1999) Problems in determining the misorientation axes, for small misorientations, using electron backscatter diffraction in the SEM. Journal of Microscopy 195, 217-225.

Raleigh C. B. and Talbot J. L. (1967) Mechanical twinning in naturally and experimentally deformed diopside. American Journal of Science 265, 151-165.

Ramsey J. G. and Huber M. I. (1983) Techniques of Modern Structural Geology. Academic Press, London.

Raterron P. and Jaoul O. (1991) High-temperature deformation of diopside single crystal: 1. Mechanical data. Journal of Geophysical Research: Solid Earth 96, 14277-14286. 
Reed S. J. B. (2005) Electron Microprobe Analysis and Scanning Electron Microscopy in Geology, 2nd ed. Cambridge University Press, Cambridge.

Roeller K. and Trepmann C. (2010). Stereo32.

Rubin A. E. (2012) Collisional facilitation of aqueous alteration of CM and CV carbonaceous chondrites. Geochim. Cosmoch. Acta 90, 181-194.

Rubin A. E., Scott E. R. D. and Keil K. (1997) Shock metamorphism of enstatite chondrites. Geochim. Cosmoch. Acta 61, 847-858.

Rubin A. E. and Swindle T. D. (2011) Flattened chondrules in the LAP 04581 LL5 chondrite: Evidence for an oblique impact into LL3 material and subsequent collisional heating. Meteoritics \& Planetary Science 46, 587-600.

Rubin A. E., Trigo-Rodriguez J. M., Huber H. and Wasson J. T. (2007) Progressive aqueous alteration of CM carbonaceous chondrites. Geochim. Cosmoch. Acta 71, 23612382.

Ruzicka A. (1990) Deformation and thermal histories of chondrules in the Chainpur (LL3.4) chondrite. Meteoritics 25, 101-113.

Scott E. R. D., Keil K. and Stoeffler D. (1992) Shock metamorphism of carbonaceous chondrites. Geochim. Cosmoch. Acta 56, 4281-4293.

Sharp T. G., de Carli P. S. and McSween H. Y. (2006) Shock Effects in Meteorites. In Meteorites and the Early Solar System II (ed. D.S. Lauretta). pp. 653-677.

Smith D. L., Ernst R. E., Samson C. and Herd R. (2006) Stony meteorite characterization by non-destructive measurement of magnetic properties. Meteoritics \& Planet. Sci. 41, 355-373.

Sneyd D. S., McSween H. Y., Jr., Sugiura N., Strangway D. W. and Nord G. L., Jr. (1988) Origin of petrofabrics and magnetic anisotropy in ordinary chondrites. Meteoritics 23, 139149. 
Stoffler D., Biscoff A., Buchwald V. and Rubin A. E. (1988) Shock effects in meteorites. In Meteorites and the Early Solar System (eds. J.F. Kerridge and M.S. Matthews). University of Arizona Press, Tucscon. pp. 165-202.

Sugiura N. and Strangway D. W. (1983) Magnetic anisotropy and porosity of chondrites. Geophysical Research Letters 10, 83-86.

Tomeoka K., Yamahana Y. and Sekine T. (1999) Experimental shock metamorphism of the Murchison CM carbonaceous chondrite. Geochim. Cosmoch. Acta 63, 3683-3703.

Tomioka N., Tomeoka K., Nakamura-Messenger K. and Sekine T. (2007) Heating effects of the matrix of experimentally shocked Murchison CM chondrite: Comparison with micrometeorites. Meteoritics \& Planet. Sci. 42, 19-30.

Tonui E., Zolensky M., Hiroi T., Nakamura T., Lipschutz M. E., Wang M.-S. and Okudaira K. (2014) Petrographic, chemical and spectroscopic evidence for thermal metamorphism in carbonaceous chondrites I: CI and CM chondrites. Geochim. Cosmoch. Acta 126, 284-306.

Trepmann C. A. (2008) Shock effects in quartz: Compression versus shear deformation, An example from the Rochechouart impact structure, France. Earth Planet. Sci. Lett. 267, 322-332.

Trigo-Rodriguez J. M., Rubin A. E. and Wasson J. T. (2006) Non-nebular origin of dark mantles around chondrules and inclusions in CM chondrites. Geochim. Cosmoch. Acta 70, 1271-1290.

Turner F. J. and Weiss L. E. (1963) Structural analysis of metamorphic tectonites. McGraw-Hill.

Weiss B. P. and Elkins-Tanton L. T. (2013) Differentiated Planetesimals and the Parent Bodies of Chondrites. Annual Review of Earth and Planetary Sciences 41, 529-560.

Woodcock N. H. (1977) Specification of fabric shapes using an eigenvalue method. Geological Society of America Bulletin 88, 1231-1236. 
Woodcock N. H. and Naylor M. A. (1983) Randomness testing in three-dimensional orientation data. Journal of Structural Geology 5, 539-548.

Yasuda M., Kitamura M. and Morimoto N. (1983) Electron microscopy of clinoenstatite from a boninite and a chondrite. Physics and Chemistry of Minerals 9, 192-196.

Zimbelman J. R. (1986) The role of porosity in thermal inertia variations on basaltic lavas. Icarus 68, 366-369.

Zolensky M., Mikouchi T., Fries M., Bodnar R., Jenniskens P., Yin Q.-z., Hagiya K., Ohsumi K., Komatsu M., Colbert M., Hanna R., Maisano J., Ketcham R., Kebukawa Y., Nakamura T., Matsuoka M., Sasaki S., Tsuchiyama A., Gounelle M., Le L., Martinez J., Ross K. and Rahman Z. (2014) Mineralogy and petrography of C asteroid regolith: The Sutter's Mill CM meteorite. Meteoritics \& Planetary Science 49, 1997-2016.

Zolensky M. E., Mittlefehldt D. W., Lipschutz M. E., Wang M.-S., Clayton R. N., Mayeda T. K., Grady M. M., Pillinger C. and Barber D. (1997) CM chondrites exhibit the complete petrologic range from type 2 to 1. Geochim. Cosmoch. Acta 61, 5099. 